\title{
Resistive switching in hafnium dioxide layers: Local phenomenon at grain boundaries
}

M. Lanza, G. Bersuker, M. Porti, E. Miranda, M. Nafría, and X. Aymerich

Citation: Appl. Phys. Lett. 101, 193502 (2012);

View online: https://doi.org/10.1063/1.4765342

View Table of Contents: http://aip.scitation.org/toc/apl/101/19

Published by the American Institute of Physics

\section{Articles you may be interested in}

Grain boundaries as preferential sites for resistive switching in the $\mathrm{HfO}_{2}$ resistive random access memory structures

Applied Physics Letters 100, 123508 (2012); 10.1063/1.3697648

Metal oxide resistive memory switching mechanism based on conductive filament properties Journal of Applied Physics 110, 124518 (2011); 10.1063/1.3671565

Conduction mechanism of $\mathrm{TiN} / \mathrm{HfO}_{\mathrm{X}} / \mathrm{Pt}$ resistive switching memory: A trap-assisted-tunneling model Applied Physics Letters 99, 063507 (2011); 10.1063/1.3624472

Resistive switching phenomena: A review of statistical physics approaches Applied Physics Reviews 2, 031303 (2015); 10.1063/1.4929512

Resistive switching in silicon suboxide films Journal of Applied Physics 111, 074507 (2012); 10.1063/1.3701581

Reproducible switching effect in thin oxide films for memory applications Applied Physics Letters 77, 139 (2000); 10.1063/1.126902

\section{Scilight} Sharp, quick summaries illuminating the latest physics research

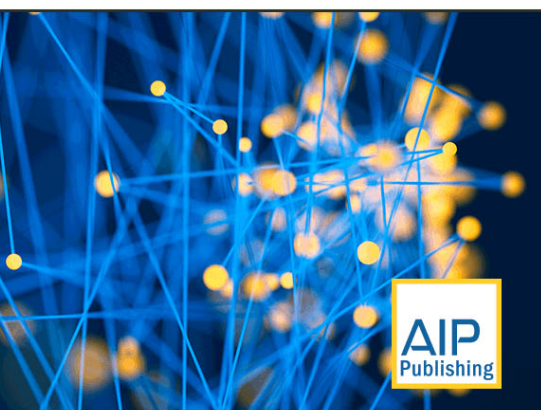




\title{
Resistive switching in hafnium dioxide layers: Local phenomenon at grain boundaries
}

\author{
M. Lanza, ${ }^{1, a)}$ G. Bersuker ${ }^{2}$ M. Porti, ${ }^{1}$ E. Miranda,${ }^{1}$ M. Nafría, ${ }^{1}$ and X. Aymerich ${ }^{1}$ \\ ${ }^{1}$ Departament d'Enginyeria Electrònica, Universitat Autònoma de Barcelona, Edifici Q, \\ 08193 Bellaterra, Spain \\ ${ }^{2}$ SEMATECH, Austin, Texas 78741, USA
}

(Received 21 April 2012; accepted 18 October 2012; published online 6 November 2012)

\begin{abstract}
Overcoming challenges associated with implementation of resistive random access memory technology for non-volatile information storage requires identifying the material characteristics responsible for resistive switching. In order to connect the switching phenomenon to the nano-scale morphological features of the dielectrics employed in memory cells, we applied the enhanced conductive atomic force microscopy technique for in situ analysis of the simultaneously collected electrical and topographical data on $\mathrm{HfO}_{2}$ stacks of various degrees of crystallinity. We demonstrate that the resistive switching is a local phenomenon associated with the formation of a conductive filament with a sufficiently small cross-section, which is determined by the maximum passing current. Switchable filament is found to be formed at the dielectric sites where the forming voltages were sufficiently small, which, in the case of the stoichiometric $\mathrm{HfO}_{2}$, is observed exclusively at the grain boundary regions representing low resistant conductive paths through the dielectric film. (C) 2012 American Institute of Physics. [http://dx.doi.org/10.1063/1.4765342]
\end{abstract}

Resistive random access memory (RRAM) technology is promising for a variety of non-volatile information storage applications since it can be implemented using simple crossbar structures, ${ }^{1,2}$ and is characterized by high switching speed and excellent retention and scalability. ${ }^{3-5}$ The principle of operation of bipolar RRAMs involves a change in the current through the dielectric in a metal-insulator-metal (MIM) structure by alternative applications of biases of opposite polarity. ${ }^{1}$ In one of the most promising classes of transition metal oxides considered for RRAM implementation-fab-friendly $\mathrm{HfO}_{2}$-based dielectrics ${ }^{6}$ - the switching mechanism is believed to be based on a change in the resistivity of a conductive filament (CF), which needs to be initially formed in the insulator. ${ }^{7}$ However, the nature of the filament for resistive switching (RS) in these materials has never been experimentally demonstrated. Until now RS was observed only in lithographically fabricated devices (MIM capacitors or transistors), the gate areas of which are covered by a metal film, which prevents a direct observation of the post-forming features of the dielectric material. ${ }^{8,9}$ Therefore, a device-level study cannot unambiguously address the questions of (a) whether the switching is filament-driven and (b) which morphological properties of the dielectric can be responsible for the RS. In this study, the RS phenomenon in $\mathrm{HfO}_{2}$ dielectric, in both the amorphous and polycrystalline phases, was studied at the nanoscale using conductive atomic force microscopy (CAFM). The high lateral resolution of the CAFM measurements $(\sim 10 \mathrm{~nm})$ allows isolating the RS associated with a single conductive filament. This study therefore had two goals: (1) to verify the local nature of the filament responsible for the resistive switching, and (2) to identify the dielectric morphological features responsible for switching. The latter allows for link-

\footnotetext{
${ }^{\text {a) }}$ Author to whom correspondence should be addressed. Electronic mail: mario.lanza@uab.cat. Telephone: +34935868463. FAX: +34935812600.
}

ing atomic-level structural characteristics of the material to their electrical properties.

The sample set included $\mathrm{HfO}_{2} / 3 \mathrm{~nm} \quad \mathrm{Zr} / 10 \mathrm{~nm}$ TiN/40 nm TaN stacks with amorphous (sample A: $3 \mathrm{~nm}$ thick, no anneal) and polycrystalline (sample B: $3 \mathrm{~nm}$ thick and sample C: $6 \mathrm{~nm}$, both annealed at $400^{\circ} \mathrm{C}$ during $30 \mathrm{~s}$ ) $\mathrm{HfO}_{2}$ films. $\mathrm{HfO}_{2}$ stacks were created by atomic layer deposition, and the phase change was induced by annealing in $\mathrm{N}_{2}$ atmosphere at $400^{\circ} \mathrm{C}$ during $30 \mathrm{~s}$, as corroborated by extended $\mathrm{x}$-ray absorption fine structure (EXAFS) measurements. ${ }^{10,11}$ When scanning the surface of each sample with the Pt-Ir coated Si AFM tip (from Bruker, model SCM-PIT), the system was isolated in a high vacuum chamber at $10^{-7}$ Torr, which reduces tip-sample effective contact area down to $10 \mathrm{~nm}^{2}$. The nominal current-limited voltage scans were made using the Keithley 2400 as the voltage source. Large current dynamic range I-V curves were obtained with an Enhanced Atomic Force Microscope (ECAFM), ${ }^{12}$ which basically consists of an Agilent 4541B semiconductor parameter analyzed (SPA) connected to the electronics of the CAFM. This setup has three main advantages: (1) the possibility to apply voltages much larger than using a standard CAFM, (2) the ability to apply a current-limited bias, and (3) a wide current dynamic range (1 pA-1 mA, which is necessary to measure RS).

First, the morphological properties of the different as-grown $\mathrm{HfO}_{2}$ films (that is, prior to electrical stress) were analyzed using the collected topographical maps (Figs. 1(a)1(c), the RMS value of the image is also indicated). In no one of all collected images, a perfect granular structure, which is characteristic of the polycrystalline samples, can be observed. This is consistent with the previous reports ${ }^{13,14}$ showing that the grains in polycrystalline samples can be distinguished in topographic maps only when the annealing temperature is sufficiently high. However, an increase in RMS points to the anneal-induced phase modification, which 


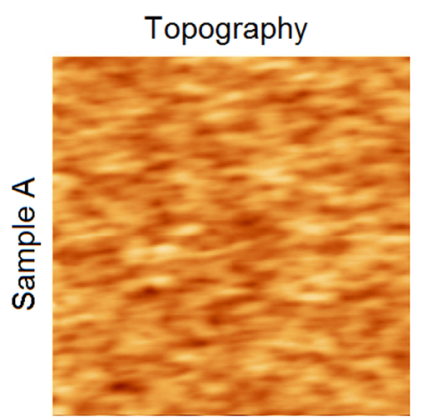

(a) $\mathrm{RMS}=2.19 \mathrm{~nm}$

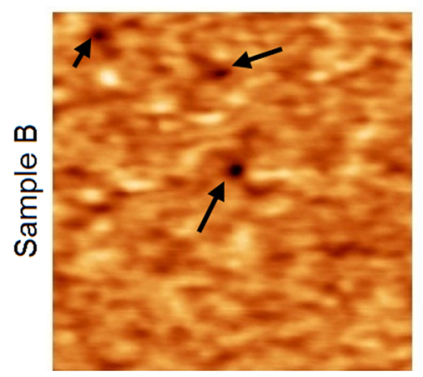

(b) $\mathrm{RMS}=4.62 \mathrm{~nm}$

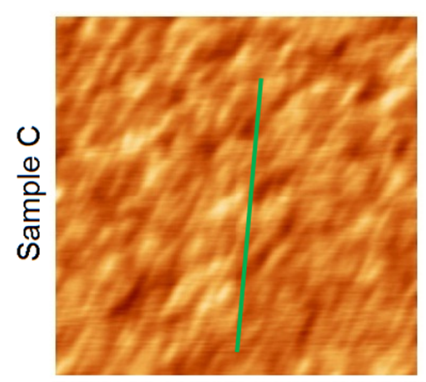

(c) $\mathrm{RMS}=6.53 \mathrm{~nm}$

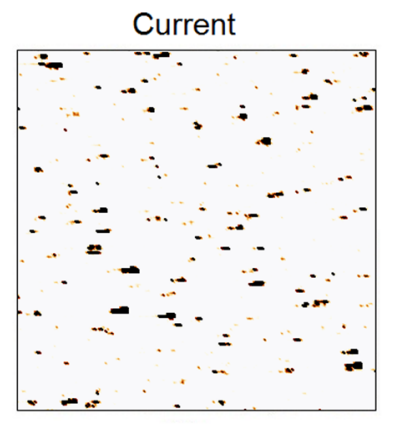

(d) $\mathrm{RMS}=9.93 \mathrm{pA}$

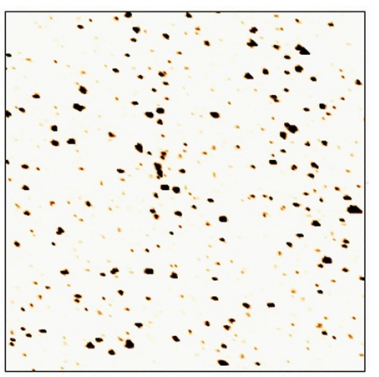

(e) $\mathrm{RMS}=16.15 \mathrm{pA}$

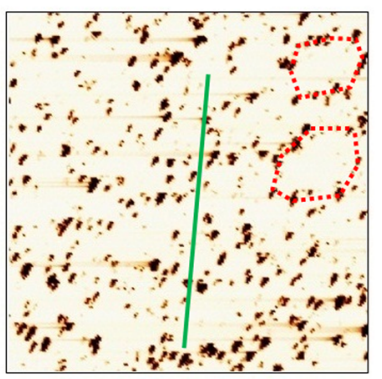

(f) $\mathrm{RMS}=23.97 \mathrm{pA}$
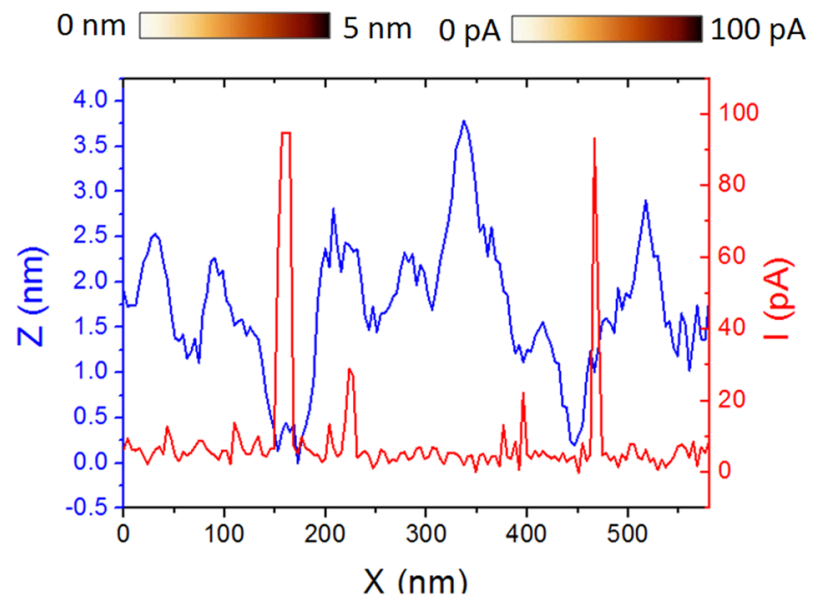

(g)

FIG. 1. Topographic and current maps obtained (simultaneously) on sample A (non-annealed $3 \mathrm{~nm} \mathrm{HfO}_{2}$, (a) and (d)), sample B (annealed $3 \mathrm{~nm} \mathrm{HfO}_{2}$, (b) and (e)), and sample $\mathrm{C}$ (annealed $6 \mathrm{~nm} \mathrm{HfO}_{2}$, (c) and (f)) by applying a bias of $1 \mathrm{~V}, 1.4 \mathrm{~V}$, and $2.6 \mathrm{~V}$, respectively (identical electrical fields). All images are $1 \mu \mathrm{m} \times 1 \mu \mathrm{m}$. The arrows in (b) indicate surface modification and the dashed lines in (f) outline nanocrystals. (g) Topographic and current scans performed along the solid line shown in (c) and (f).

was actually corroborated by EXAFS in Refs. 10 and 11 . To clarify the crystalline phase of each sample, we obtained complementary information from the current scans. Figures 1(d)-1(f) show the current scans data collected simultaneously with that of the topographic maps in Figs. 1(a)-1(c), where the higher leakage current sites are present. In the current maps, the phase modification can be deduced based on the distribution of the leaky spots. In the case of the amorphous sample (sample A), the leaky sites are randomly spread over the whole measured area and might be related to local changes in the dielectric thickness and/or the presence of defects. ${ }^{13,15}$ On the contrary, the current map of the thicker sample (sample C) exhibits not only larger dispersion (higher RMS) but also a well-defined granular pattern (Fig. 1(f), red dashed line), which can be assigned to the sample's polycrystalline structure (in agreement with the EXAFS data $^{10,11}$ ). Note that, even if (as mentioned) the topographic images do not show perfect granular patterns, in some cases the correlation between topography and current can be observed. The section highlighted in Figs. 1(c) and 1(f) is shown in Fig. 1(g). Note that, higher currents observed at certain locations (Fig. 1(f)) are associated with a significant thickness reduction, suggesting that these locations correspond to grain boundaries (GBs), which were shown to form depressed regions on the dielectric surface. ${ }^{14,16}$ However, it should be emphasized that leakage at GBs is not higher there only because of lower thickness but also due to a high oxygen vacancy concentration. ${ }^{17}$

In the current image of sample B (Fig. 1(e)), the granular structure is not as clear as in sample C. Accordingly, the topography (GB)-current correlation is more difficult to identify (although some depressions are seen on the surface, as indicated by the arrows and blue dashed line in Fig. 1(b), ${ }^{14,18}$ suggesting a lower level of crystallization, because the film smaller thickness (compared to sample C) impedes grain growth. ${ }^{19,20}$ In any case, the differences in the CAFM maps (Figs. 1(a), 1(b), 1(d), and 1(e)) of samples A and B, as well as the EXAFS data, ${ }^{10}$ suggest that the phase change in sample B has actually occurred.

After the as-grown samples were characterized, they were subjected to electrical stress by applying 3 consecutive voltage scans over the same area. Table I summarizes the average number and sizes of the high current spots observed in the current images measured in the 1st and 3rd scans (at the same location). In sample $A$, the number of conductive spots is seen to increase as the electrical stress (i.e., the number of scans) proceeds, but their size does not change, which suggests that the amorphous $\mathrm{HfO}_{2}$ stack is degraded due to generation of new defects at random locations. On the contrary, sample $\mathrm{C}$ does not exhibit an appreciable increase of the number of new leaky spots with longer electrical stress; however, some spots grow significantly larger suggesting progressive degradation at these locations, which coincide with the GBs. Finally, in sample B, not only the leaky spots seen during the first scan (Fig. 1(e)) become larger after the third scan but many new conductive spots are also detected. This

TABLE I. Mean amount of conductive spots and their sizes detected for all samples during the first and last current image of a sequence of three scans.

\begin{tabular}{lcccccc}
\hline \hline \multirow{2}{*}{ Magnitude } & \multicolumn{2}{c}{ Sample A } & \multicolumn{2}{c}{ Sample B } & \multicolumn{2}{c}{ Sample C } \\
\cline { 2 - 7 } & First & Third & First & Third & First & Third \\
\hline Number of spots $\left(\# / \mu \mathrm{m}^{2}\right)$ & 180 & 365 & 152 & 510 & 202 & 246 \\
Spots size $\left(\mathrm{nm}^{2}\right)$ & 144 & 155 & 84 & 115 & 122 & 183 \\
\hline \hline
\end{tabular}


could be related to the coexistence of the so called pseudoamorphous and polycrystalline phases. Thus, the data in Fig. 1 demonstrate the different modes of degradation of pseudo-amorphous and crystalline $\mathrm{HfO}_{2}$ films: In the latter, the stress-induced leakage current increase is observed mostly around the GBs.

In the next step, we addressed the RS characteristics of these films by applying ramped voltage stresses (RVS) at random locations (approx. $100 \mathrm{~nm}^{2}$ large) of each sample using an enhanced CAFM setup. ${ }^{12}$ In sample A (amorphous), large voltages (independent of polarity) were required to achieve forming on all 24 analyzed locations (Fig. 2(a)), with no RS observed in any of them. On the other hand, two distinct location types were found in sample B (Fig. 2(b)) with high forming voltages, above $12 \mathrm{~V}$ (Fig. 2(b), black squares), on most of the randomly chosen locations (16 of $19,85 \%)$ and with much lower forming voltages $\left(4<\mathrm{V}_{\mathrm{BD}}\right.$ $<6.5 \mathrm{~V}$, Fig. 2(b), red circles). At the locations with high forming voltages, no RS was observed in successive IV curves, while the low forming voltage locations exhibit typical bipolar RS behavior (Fig. 2(c)) identical to what is observed in similar MIM devices. ${ }^{21}$ Therefore, the data in Figs. 2(a)-2(c) clearly show that the RS occurs only in the $\mathrm{CFs}$ formed with sufficiently low voltages. Lower forming voltages are characteristic of GB locations due to its high density of positively charged oxygen vacancies, ${ }^{22}$ as demonstrated by Kelvin probe force microscope images, ${ }^{17}$ which were shown to support electron transport through the dielectric. $^{23,24}$ Since the surface area covered by the GBs is much smaller than that of the crystal grains, the RS is observed on only a few of the tested locations. The RVS performed with no current compliance at the RS locations (Fig. 2(d), ramp 5) resulted in a permanent dielectric breakdown (BD) at around $3.2 \mathrm{~V}$, after which no further RS was observed.

We have observed (Fig. 1) that GBs exhibit higher leakage currents and demonstrated (Fig. 2) RS at electrically leaky locations. In the next step, we performed a complete write-read-erase-read cycle measuring topographic and current maps after each operation, which allows distinguishing locations recovering their insulating properties. Sample C was used for this measurements (Fig. 3) because RS was readily observed there thanks to the sample's high degree of crystallinity. The current-limited constant voltage scans were done at different stages of the RS cycle (Figs. 3(c) and 3(e)$3(\mathrm{~g})$ ) while the topography maps before the electrical stress (Fig. 3(b)) and after the forming (or write) processes (Fig. 3(d)) were also recorded. Before electrical stress, some nanocrystals can be clearly identified (Fig. 3(b), where some of the GBs are outlined by the dashed line) even when (again, as in Fig. 1(f)) a perfect granular structure was not observed. ${ }^{13,14}$ In this scan, since no bias has been applied, no leaky spots were observed in the current map (Fig. 3(a)). When a high voltage is applied during the scan (which corresponds to a write operation, Figs. 3(c) and 3(d)), the current image (Fig. 3(c)) shows the current mostly at the GBs (dashed line). When the same area is scanned at a very low voltage (a read operation, Fig. 3(e)), the remaining leaky sites can be assigned to the CFs created by the write process. Note that although most of the CFs are concentrated at GBs (green dashed lines in Fig. 3(e)), a filament is also seen to be formed in the grain regions (a red dashed line in Fig. 3(e)), which could be related to a lateral propagation of the $\mathrm{CF}$ formed at the GB. ${ }^{14}$ Formation of this $\mathrm{CF}$ is accompanied by an apparent severe topographic distortion around this location on the dielectric surface, Fig. 3(d), which, as previously reported ${ }^{15}$ could be related not only to dielectric breakdown induced epitaxy (DBIE) but also to a significant charge trapped in the insulator in the CF region (electromigration), leading to artifacts in the topographical imaging. Afterwards, the reset process was performed by applying a negative voltage (Fig. 3(f)), and the sample was subsequently scanned with a low voltage (a read operation, Fig. $3(\mathrm{~g})$ ). Note that, despite the creation of a few new leaky spots (the red dashed line in Fig. 3(g)) caused by the high voltage in the previous
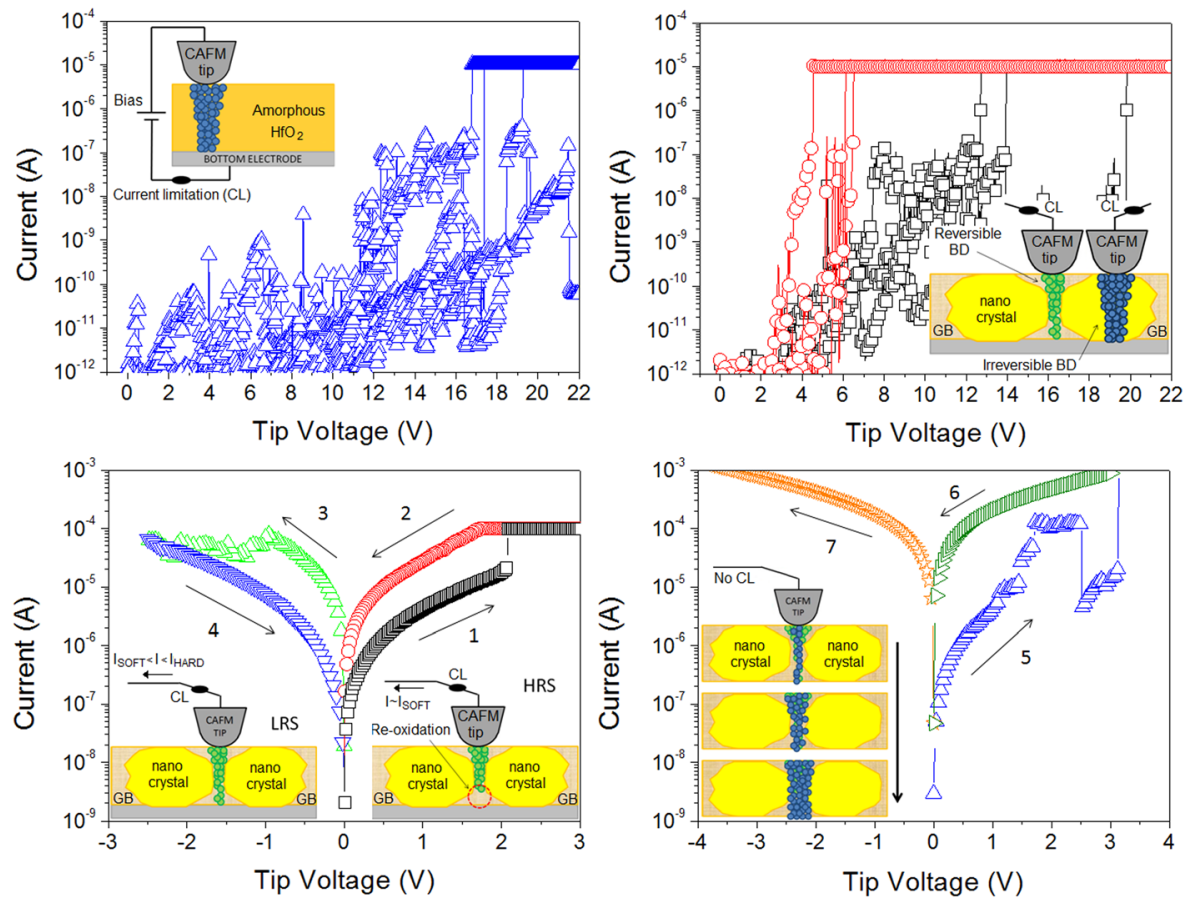

FIG. 2. Forming process at different random locations in (a) sample A (non-annealed amorphous $3 \mathrm{~nm} \mathrm{HfO}_{2}$ ) and (b) sample $\mathrm{B}$ (annealed polycrystalline $3 \mathrm{~nm} \mathrm{HfO}_{2}$ ) where two different I-V patterns associated with low voltage and high voltage formings can be distinguished. (c) An example of RS behavior observed at the low voltage forming site in (b). (d) An example of the creation of an irreversible CF at a GB location (similar to that in (c)) when the forming is done without a current compliance limit. The schematics indicate the probing location and measurement conditions. 



TOPOGRAPHY
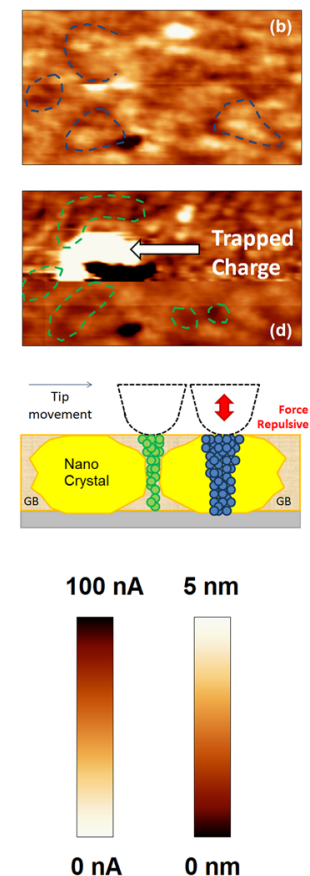

CONDUCTIVE SPOTS IN LRS

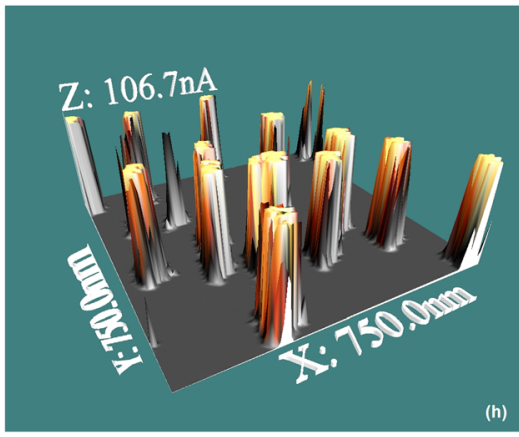

CONDUCTIVE SPOTS IN HRS

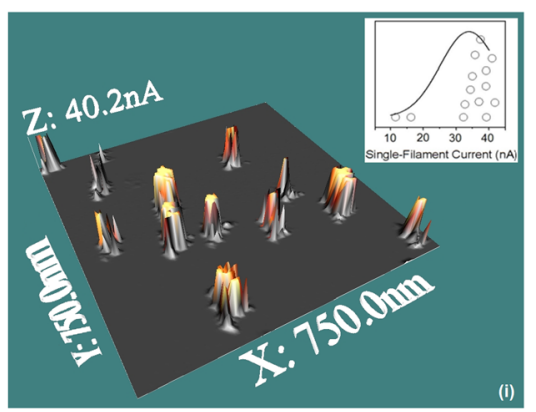

FIG. 3. (a) Current and (b) topographic images collected on the sample C (annealed, $6 \mathrm{~nm}$ $\mathrm{HfO}_{2}$ ) before forming. Current (c) and topographic (d) images collected simultaneously by applying $2 \mathrm{~V}$ scan (forming process). (e)-(g) exhibit sequential current images collected (after forming) by applying scans with the tip bias of $0.3 \mathrm{~V}$ (read), $-2 \mathrm{~V}$ (reset), and $0.3 \mathrm{~V}$ (read), respectively (absolute values of the currents are plotted). During the forming process (c), the current compliance was $1 \mu \mathrm{A}$ and the rest of the current images were obtained without current limitations. All images were taken on the same $550 \mathrm{~nm} \times 300 \mathrm{~nm}$ area. The schematic illustrates the probing locations where the CFs were created in panel (d), that is, through both GB and nanocrystals. (h) and (i) show the read currents in multiple filaments switched between LRS and HRS before and after the first reset. scan (resulting in uncontrolled forming at these locations), Fig. 3(g) clearly shows a dramatic reduction of the current at those highly conductive spots (CF), which were formed at the GBs during the write operation (the green dashed line in Fig. $3(\mathrm{~g}))$. On the other hand, a reset operation could not recover the insulating properties of the CFs formed (at much higher voltages) at the grain sites (the red dashed line in Fig. $3(\mathrm{~g})$ ), indicating that at those locations the CFs are irreversible. This result, which agrees with the data in Figs. 2(b) and 2(c), indicates that a region of the irreversible forming is associated with an unusually large amount of positive charge in the dielectric (fixed charges), which might be induced as a result of metallization of this region by the metal ions diffused from the CAFM probe tip (note that the metallic coating of the Pt-Ir tip wears out under high current density conditions). On the contrary, the reversible CFs might be associated with the filaments formed by the oxygen vacancies, which can be oxidized during the reset process leading the filament switching to a higher resistive state. ${ }^{24}$

Finally, to obtain a statistically meaningful data set on the reversible CFs, the write-read-erase-read sequence (Figs. $3(\mathrm{a})-3(\mathrm{~g}))$ was repeated on several areas of the sample C, and the created CFs were analyzed. As an example, Figs. 3(h) and 3(i) display the current maps collected during the read scan after the set (LRS) and reset (HRS) operations performed on a random area in sample C. A statistical analysis shows that while the maximum LRS current is determined by the current saturation level (100 nA) available in the given setup, in the HRS, the average current is reduced to $33.87 \pm 8.42 \mathrm{nA}$ (inset in Fig. 3(i)).

By using the CAFM tip as the metal electrode in a MIM structure, it was in situ demonstrated that the RS in $\mathrm{HfO}_{2}$-based resistive memory structures is associated with a nanometer sized CF. The results indicate that the resistive switching behavior observed in pseudo-amorphous and polycrystalline $\mathrm{HfO}_{2}$ stacks is related to conductive filaments located at the grain boundaries. At GBs, the forming voltages are found to be essentially lower than those at the grain sites where no RS is observed.

This work has been partially supported by the Ministerio de Economia y Competitividad of Spain (TEC2010-16126) and the Generalitat de Catalunya (2009SGR-78).

${ }^{1}$ R. Waser, R. Dittmann, G. Staikov, and K. Szot, Adv. Mater. 21, 26322663 (2009).

${ }^{2}$ S. H. Chang, S. B. Lee, D. Y. Jeon, S. J. Park, G. T. Kim, S. M. Yang, S. C. Chae, H. K. Yoo, B. S, Kang, M. J. Lee, and T. W. Noh, Adv. Mater. 23, 4063-4067 (2011).

${ }^{3}$ C. Rohde, B. J. Choi, D. S. Jeong, S. Choi, J. S. Zhao, and C. S. Hwang, Appl. Phys. Lett. 86, 262907 (2005).

${ }^{4}$ C. Rossel, G. I. Meijer, D. Bremaud, and D. Widmer, J. Appl. Phys. 90, 2892 (2001).

${ }^{5}$ I. G. Baek, M. S. Lee, S. Seo, M. J. Lee, D. H. Seo, D. S. Suh, J. C. Park, S. O. Park, H. S. Kim, I. K. Yoo, U. I. Chung, and J. T. Moon, in Technical Digest-International Electron Devices Meeting (IEEE, 2004), p. 587.

${ }^{6}$ S. Lee, W. G. Kim, S. W. Rhee, and K. Yong, J. Electrochem. Soc. 155(2), H92-H96 (2008).

${ }^{7}$ S. J. Choi, G. S. Park, K. H. Kim, S. Cho, W. Y. Yang, X. S. Li, J. H. Moon, K. J. Lee, and K. Kim, Adv. Mater. 23, 3272-3277 (2011).

${ }^{8}$ S. Long, C. Cagli, D. Ielmini, M. Liu, and J. Suñe, IEEE Electron Device Lett. 32, 1570-1572 (2011).

${ }^{9}$ Z. Yan, Y. Guo, G. Zhang, and J. M. Liu, Adv. Mater. 23, 1351-1355 (2011).

${ }^{10}$ P. S. Lysaght, J. C. Woicik, M. A. Sahiner, S. C. Song, B. H. Lee, and R. Jammy, J. Non-Cryst. Solids 354(2-9), 399-403 (2008).

${ }^{11}$ P. S. Lysaght, J. C. Woicik, M. A. Sahiner, B. H. Lee, and R. Jammy, Appl. Phys. Lett. 91, 122910 (2007).

${ }^{12}$ X. Blasco, M. Nafria, and X. Aymerich, Rev. Sci. Instrum. 76, 016105 (2005).

${ }^{13}$ J. Pétry, W. Vandervorst, O. Richard, T. Conard, P. DeWolf, V. Kaushik, A. Delabie, and S. Van Elshocht, in Integration of Advanced Micro-and Nanoelectronic Devices-Critical Issues and Solutions, edited by J. Morais, D. Kumar, M. Houssa, R. K. Singh, D. Landheer, R. Ramesh, R. M. Wallace, S. Guha, and H. Koinuma (Mater. Res. Soc. Symp. Proc., 2004), Vol. 811, pp. D6.10.01-D6.10.06.

${ }^{14}$ V. Iglesias, M. Porti, M. Nafría, X. Aymerich, P. Dudek, T. Schroeder, and G. Bersuker, Appl. Phys. Lett. 97, 262906 (2010).

${ }^{15}$ M. Porti, M. Nafria, M. C. Blum, X. Aymerich, and S. Sadewasser, Appl. Phys. Lett. 81, 3615-3617 (2002). 
${ }^{16} \mathrm{G}$. Bersuker, J. Yum, L. Vandelli, A. Padovani, L. Larcher, V. Iglesias, M. Porti, M. Nafría, K. McKenna, A. Shluger, P. Kirsch, and R. Jammy, Solid-State Electron. 65-66, 146-150 (2011).

${ }^{17}$ V. Iglesias, M. Lanza, K. Zhang, A. Bayerl, M. Porti, M. Nafría, X. Aymerich, G. Benstteter, Z. Y. Shen, and G. Bersuker, Appl. Phys. Lett. 99, 103510 (2011).

${ }^{18}$ L. Aguilera, W. Polspoel, A. Volodin, C. Van Haesendonck, M. Porti, W. Vandervorst, M. Nafria, and X. Aymerich, in International Reliability Physics Symposium (IEEE, 2008), pp. 657-658.

${ }^{19}$ V. Cosnier, P. Besson, V. Loup, L. Vandroux, S. Minoret, M. Cassé, X. Garros, J. M. Pedini, S. Lhostis, K. Dabertrand, C. Morin, C. Wiemer, M. Perego, and M. Fanciulli, Microelectron. Eng. 84, 1886-1889 (2007).
${ }^{20}$ F. Bohra, B. Jiang, and J. M. Zuo, Appl. Phys. Lett. 90, 161917 (2007).

${ }^{21}$ G. Bersuker, D. C. Gilmer, D. Veksler, J. Yum, H. Park, S. Lian, L. Vandelli, A. Padovani, L. Larcher, K. McKenna, A. Shluger, V. Iglesias, M. Porti, M. Nafría, W. Taylor, P. D. Kirsch, and R. Jammy, in Technical Digest-International Electron Devices Meeting (IEEE, 2010), pp. 456459.

${ }^{22}$ K. McKenna and A. Shluger, Appl. Phys. Lett. 95, 222111 (2009).

${ }^{23}$ L. Vandelli, A. Padovani, L. Larcher, R. G. Southwick III, W. B. Knowlton, and G. Bersuker, IEEE Trans. Electron Devices 58, 2878-2887 (2011).

${ }^{24}$ G. Bersuker, D. C. Gilmer, D. Veksler, P. Kirsch, L. Vandelli, A. Padovani, L. Larcher, K. McKenna, A. Shluger, V. Iglesias, M. Porti, and M. Nafria, J. Appl. Phys. 110, 124518 (2011). 